\title{
Impact of MELD allocation system on the outcomes of deceased donor liver transplantation: a single-center experience
}

Jeong-Moo Lee, Han Sang Park, Kwangpyo Hong, Eui Soo Han, Suk Kyun Hong, Nam-Joon Yi, Kwang-Woong Lee, Kyung-Suk Suh

Division of Hepatobiliary, Department of Surgery, Seoul National University Hospital, Seoul, Korea

Background: The model for end-stage liver disease (MELD)-based allocation system replaced the Child-Turcotte-Pugh (CTP) score-based system for organ allocation of the liver in Korea. The aim of this study is to analyze the changes of outcomes and to describe arising issues before and after the MELD system.

Methods: From June 2014 to June 2018, 129 patients were selected from recipients who underwent deceased donor liver transplantation (DDLT) in Seoul National University Hospital. Pediatric cases were excluded. Patients were divided into two groups according to the allocation system (52 in the MELD group, 77 in the CTP group).

Results: The MELD score of the two groups differed significantly (37.8 \pm 2.0 in the MELD group, $31.0 \pm 8.2$ in the CTP group, $\mathrm{P}=0.001)$. The etiology of patients was changed the difference in etiology for liver transplantation, proportion of alcoholic cirrhosis is increased in the era of MELD allocation system. However, the proportion of hepatitis B related liver cirrhosis and hepatocellular carcinoma were decreased. Long-term survival rate in CTP group was $80.1 \%$ but it was decreased to $75 \%$ in MELD group. There were no differences of the complication rate in the CTP group and MELD group (35\% vs. $31 \%$ ).

Conclusions: The MELD allocation system distributes the liver to severely ill patients, resulting in poor performance after surgery, and as proportion of alcoholic cirrhosis increase, problems such as re-drink failure may become an issue in the future. It is necessary to adjust MELD allocation system for increasing outcomes after DDLT.

Corresponding author: Jeong-Moo Lee

E-mail: jmleetpl@gmail.com

(c) The Korean Society for Transplantation

This is an Open Access article distributed under the terms of the Creative Commons Attribution Non-Commercial License (http://creativecommons.org/licenses/by-nc/4.0/) which permits unrestricted non-commercial use, distribution, and reproduction in any medium, provided the original work is properly cited. 